\title{
O.R.C.- Processed Au Electrode towards Dual Signal Enhancement by Polishing Treatment
}

\author{
Chih-Hsien Hsu', Chia-Ming Yang ${ }^{1,2,3,5}$, Ching-Hsiang Chen ${ }^{8}$, Yu-Jen Lu' ${ }^{6}$ Chao-Sung Lai*1,4,7
}

${ }^{1}$ Department of Electronic Engineering, Chang Gung University, Taiwan 259 Wen-Hwa 1st Road, Kweishan 333, Taoyuan, Taiwan

E-mail: *cslai@mail.cgu.edu.tw

${ }^{2}$ Institute of Electro-Optical Engineering, Chang Gung University, Taoyuan, Taiwan

${ }^{3}$ Biosensor Group, Biomedical Engineering Research Center, Chang Gung University, Taoyuan, Taiwan

${ }^{4}$ Department of Nephrology, Chang Gung Memorial Hospital, Taiwan

${ }^{5}$ Department of General Surgery, Chang Gung Memorial Hospital, Linkou, Taiwan

${ }^{6}$ Department of Neurosurgery, Chang Gung Memorial Hospital Linkou Medical Center and College of Medicine

${ }^{7}$ Department of Materials Engineering, Ming Chi University of Technology, Taiwan

${ }^{8}$ Sustainable Energy Development Center, National Taiwan University of Science and Technology, Taiwan

\begin{abstract}
The fabrication of 3D gold nanoparticle layer was processed by O.R.C. (oxidation and reduction cycle) and detection of protein via dual signal model (Raman Spectroscopy and Electrochemical Study). In this study, it is demonstrated that the gold electrode with gold nanopaticle layers may serve as excellent SERS substrates in chemical and biomedical detection. In this work, we performed polishing technology to optimize the O.R.C. process and demonstrated the important correlation between polishing technology and nanoparticle layer substrates in Raman signal mapping mode. Overall, results indicated that the method with polishing treatment for dual signal model led to signal improvement and can be potentially used to develop environmental pollution and biomedical sensing platform.
\end{abstract}

Key words: Raman Spectroscopy, Gold nanoparticle, Electrochemical sensor, Oxidation and Reduction Cycle, Polishing Technology

\section{INTRODUCTION}

Creation of metal nanostructure technology is a common method in Surface Enhanced Raman Scattering (SERS) and Electrochemical Sensor applications [1]. There are different types of sensing signal model combining Raman technique with optical scattering signal and another surface potential difference on substrate for Electrochemical Sensor. It means the building of nanostructures surface is a significant process towards direct contact with the molecule interaction for signal generation. In fact, increase in the surface area and conductivity of electrochemical sensor contribute to the optimization of signal, whereas, the SERS effect from localized surface plasma resonance (LSPR) for generation of hot spots is produced when specific shape is formed on the surface [2].

In our method, an optimization technique is offered by polishing gold electrode to obtain similar surface area to uniform nanoparticle layer on the sensing area. This method has been proven to enable the detection of protein and chemical materials using Raman spectrum and cyclic voltammetry (CV) curve, respectively. Moreover, Raman signal can be measured quickly with no damage before the electrochemical testing. Finally, the optimized dual signal setup that combines fast screening of SERS and electrochemical measurement provide double confirmation which hold potency for clinical uses in the future point of care.

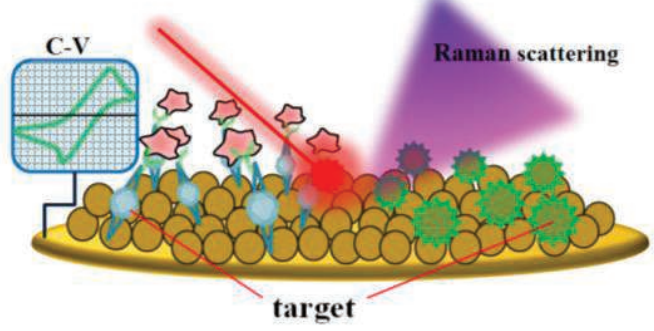

Fig. 1. shows the dual signal measurement of the actual situation. 


\section{EXPERIMENT}

The gold electrode were ground with machine using different sizes of Alumina polish for polishing and went through a 10 min ultrasonic oscillation to ensure smooth and clean surface. The O.R.C. process used the conventional three electrode system to expose the gold electrode to redox cycle. Fig.1 shows the measurement of the actual situation. Then, the target was linked or deposited on the surface by droplets and stood for natural drying. The Raman measurements of the gold substrates were carried out on a Raman spectrometer (UniDRON) with the excitation wavelength of $785 \mathrm{~nm}$. CV measurements were recorded between -0.05 and $0.4 \mathrm{~V}$ at a scan rate of 50 $\mathrm{mV} / \mathrm{s}$.

\section{RESULTS AND DISCUSSION}

In the first experiment, the current decreased when the protein immobilized onto the gold surface as recorded by of CV measurements (fig. 2). It is because the surface potentials changed when the protein (Beta Amyloid [1-42]) occupied the gold surface. As seen in Fig 3, it is observed that this configuration resulted a great SERS response in the detection of R6G(-10M) and anti-body(Beta-Amyloid [1-28]), shown by the unique fingerprint peaks on the spectra. Fig 4. shows SEM images of the proposed substrate. It is displayed that nanoparticle layers become denser and more uniform with polishing treatment on the surface. Fig 5 reveals that the $\mathrm{R} 6 \mathrm{G}(-4 \mathrm{M})$ Raman signal intensity is more uniform due to intense nanoparticle formation, shown at Raman shift of $\sim 1500 \mathrm{~cm}^{-1}$ (Raman mapping area is 1000um ${ }^{2}$, 400points).

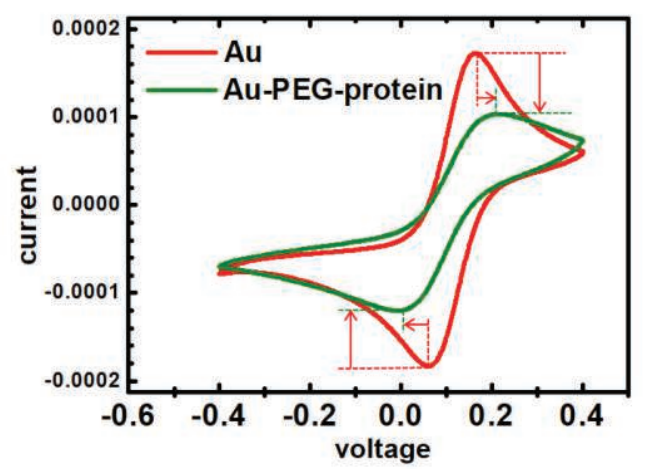

Fig. 2. Cyclic voltammogram (C-V) (red) Au electrode and (green)Au-PEG-protein biosensors.

\section{References}

[1] Dongdong Lin et al., Large-Area AuNanoparticle-Functionalized Nanorod Arrays for Spatially Uniform Surface Enhanced Raman Spectroscopy, ACS

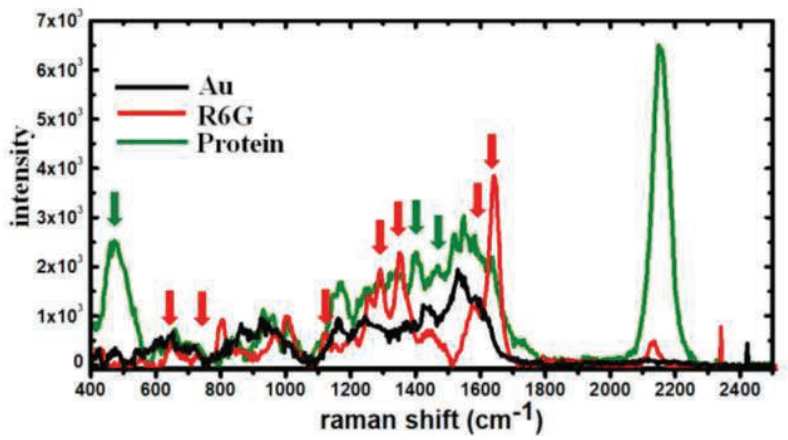

Fig. 3.Raman peaks response of (black)Au, (red) R6G,(green)Protein

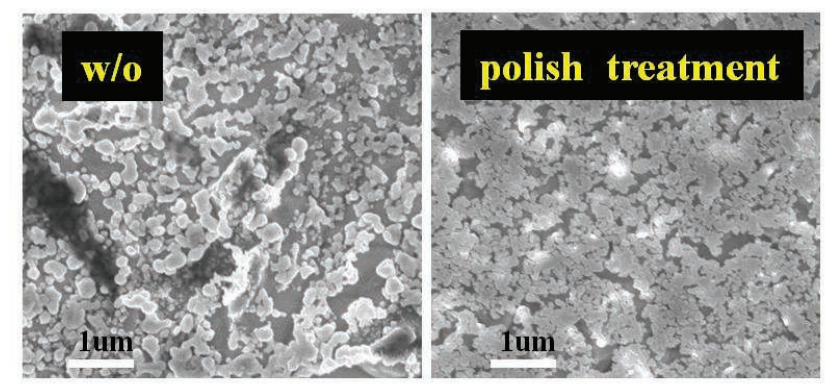

Fig. 4.SEM figure of gold surface after the polish treatment and $w / 0$.

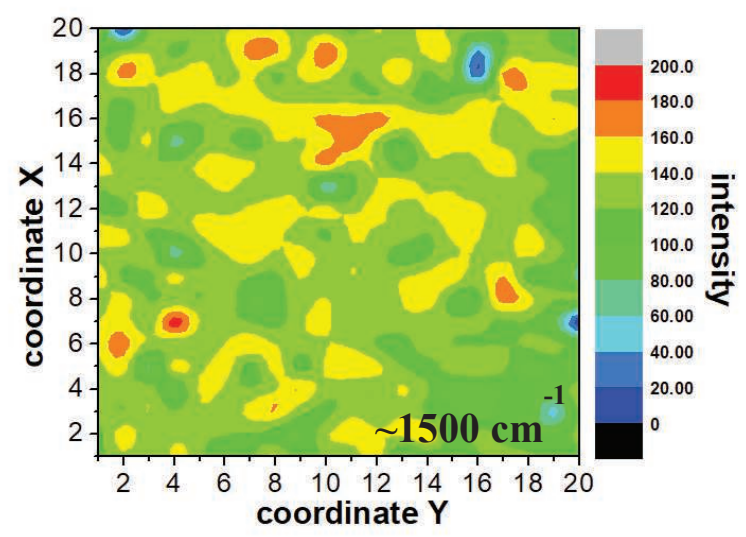

Fig.5. Raman mapping response of R6G signal (Raman shift of $1500 \mathrm{~cm}-1$ )

\section{Conclusion}

This study demonstrates the development of highly sensitive process for the detection of protein and R6G using dual signal model. Unique gold electrode properties treated with polishing technique to control particle density for surface treatment was successfully verified. Overall, this method is cost effective, highly sensitive and stable for chemical and biomedical applications.

\section{Nano , 2017, 11, 1478-1487, DOI:} 10.1021/acsnano.6b06778

[2] LIANG-YIH CHEN et al., Nanoarchitecture's Influence on Surface-Enhanced Spectroscopy, IEEE NANOTECHNOLOGY MAGAZINE, 2017 1932-4510/1 